\title{
Virtual Surgery System Using Deformable Organ Models and Force Feedback System with Three Fingers
}

\author{
Naoki SUZUIKI ', Asaki HATTORI '. Akihiro TAKATSU ', Takahiro KUMANO², Akio IKEMOTO², \\ Yoshitaka ADACHI ${ }^{2}$, Akihiko UCHIYAMA ${ }^{3}$ \\ 'Institule for High Dimensional Medical Imaging, Jikei University School of Medicine \\ 4-11-1, Izumi honcho, Komae-shi, Tokyo 201-8601, Japan \\ nsuzuki@jikci.ac.jp, hat@ikd.att.ne.jp. takatsu@jikci.ac.jp \\ 'Suzuki Motor Corporation R \& DCenter \\ 2-1, Sakura-namiki, Tsuzuki-ku, Yokohama, Kanagawa 224-0046, Japan \\ \{kumano, ikemoto, adachi\}@yrd.suzuki.co.jp \\ 'School of Science and Engineering. Waseda University \\ 3-4-1, Okubo, Shinjuku-ku, Tokyo 169, Japan \\ uchiyama@uchiyama.comm.waseda.ac.jp
}

\section{Abstract}

We aimed to develop a virtual surgery system capable of performing surgical mancuvers on elastic organs, the structure of which has been obtained from a patient. We tried to manipulate three dimensional (3D) organs as elastic models by hand or surgical tools in real time in a virtual cnvironment. And we tried to obtain the sense of touch by using force feedback device with manipulator attached to the thumb, forefinger and middle finger of the operator.
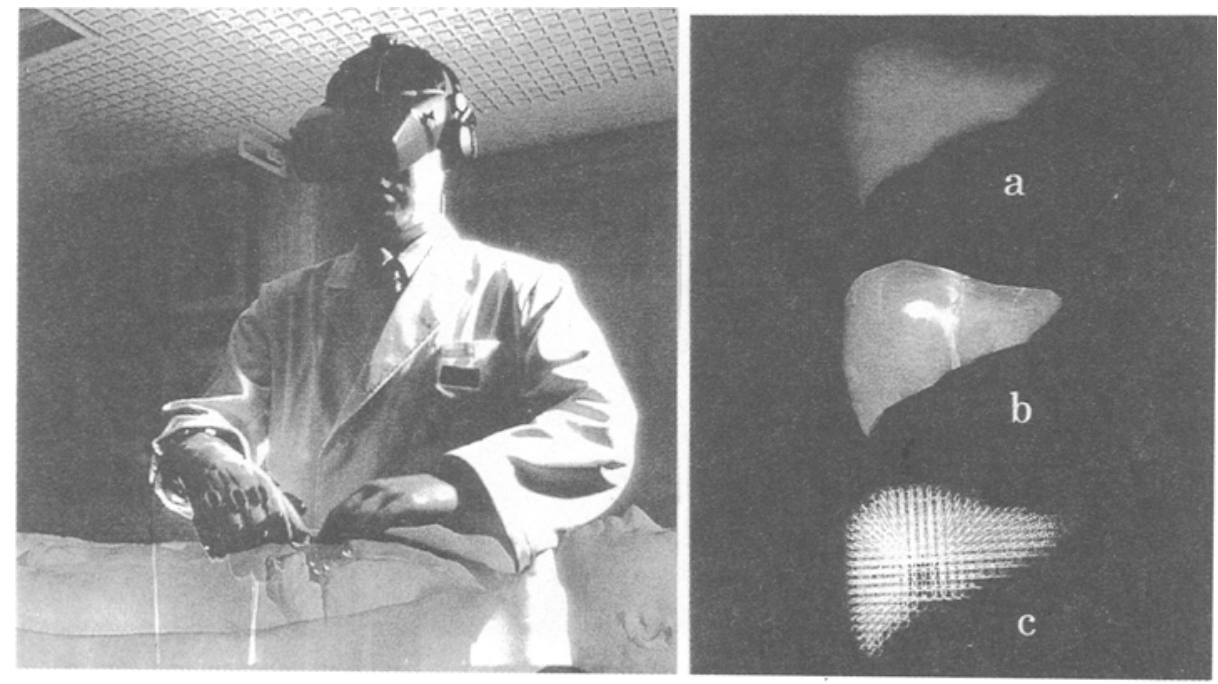

Fig.1.(upper left) A typical scene of the operator performing a surgical simulation.

Fig. 2.(upper right) Structure of the deformable organ model of a liver. Fig. $2 b$ is the reconstructed liver with a texture mapping of autopsied liver tissue. Fig. $2 \mathrm{c}$ is inner structure of the reconstructed liver model. The liver model was filled with 1880 spheres. 


\section{Method}

\subsection{R surgery simulation system}

We wanted to devise a system that would allow pushing, pinching, or incising of soft tissue models in the surgical simulation. When a large incision is made, the inner structures of an organ, such as blood vessels, are also likely to be incised or deformed. Our system therefore had the following requirements. At first, the simulation should performed as real time simulation and the image should be real. That is, the system have to represent the accurate and detailed shape of the patient's organ and to deform the organ according to physical and anatomical knowledge. Second, the organ model should be performed quantitative deformation with accuracy including the organ's inner structures. In the real time simulation, user is able to manipulate (push, pick, pinch, incise and excise) the organ model by hand or with surgical tools in virtual space. And we need to devise a system to connect casily to the force feedback device and to obtain values for force feedback.

The image generation in our system is performed by an Onyx Reality Engine2 (Silicon Graphics Cray Inc). We thought that it is difficult at present to perform the calculations of organ structure with the compiled anatomical structure for the finite element method in real time due to speed limits of the processor. It is also difficult to divide human structures automatically into finite elements. We have proposed a so-called "sphere-filled model" to produce an elastic organ model. In this model, a mass composed of small spheres fills the inside of a 3D organ model. Organ models are reconstructed from contour organ sets oblained from 3D data sets using CT or MRI. We fill the inside of the organ with some element spheres. All element spheres have the same radius, and arc placed at a face-centered cubic lattice. The external force applied to the organ model displaces some of these spheres, which in turn displace the spheres surrounding them until ul timately the shape of the entire organ is deformed. The magnitude and direction of the force generated when the spheres are moved, determines their return to their original position, allowing the force-feedback system to generate a sense of touch.

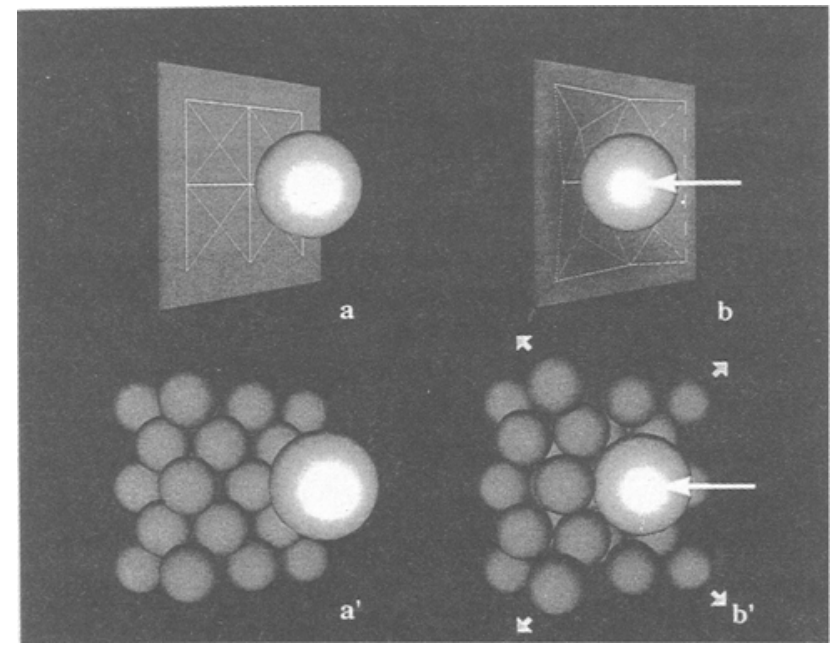

Fig.3. The basic behavior of model deformation. If external force is applied to the model, it is deformed by the movement of each element sphere. 


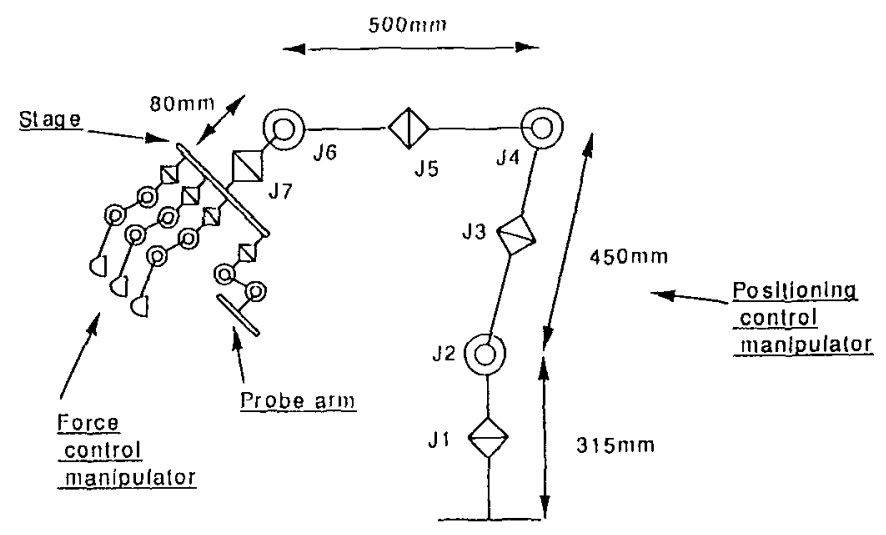

Fig.4. The block diagram of the design of the force feedback device system. The locations of joints and relational location of motion controlled manipulators are seen in the schema.

\section{2-2. Force feedback device}

We tried to develop a force feedback device which gives the user the sense of touch as if actual contact were being made with the organ. However, in the field of surgical simulation, there are some important points to be considered when a force feedback device is designed. One is how to measure the movement of the operator's hand with high accuracy. Another is how to give the operator's hand a realistic sensation of the virtual environment. To satisfy these requirements, the development of a high performance force feedback system is anticipated. We designed and built a sixteen degrees-offreedom (DOF) force feed back device for manual interaction with virtual environments .

The features of the device manufactured for our virtual surgery system are summarized as follows. At first, the force feedback system is composed of two types of manipulators, a force control manipulator and a motion control manipulator. And we tried to design three force control manipulators, those are attached to the end of the motion control manipulator. Second, both ends of each force control manipulator are attached to the thumb, forefinger and middle finger of the operator. In addition, the force control manipulator has a joint structure with minimal inertia and less friction. And the motion control manipulator has a mechanical stiffness should be realized in the system to complete surgical maneuvers without stress. Fig. 4 shows the block diagram of the design of the force feedback device system. The locations of joints and relational location of motion controlled manipulators are seen in the schema. 


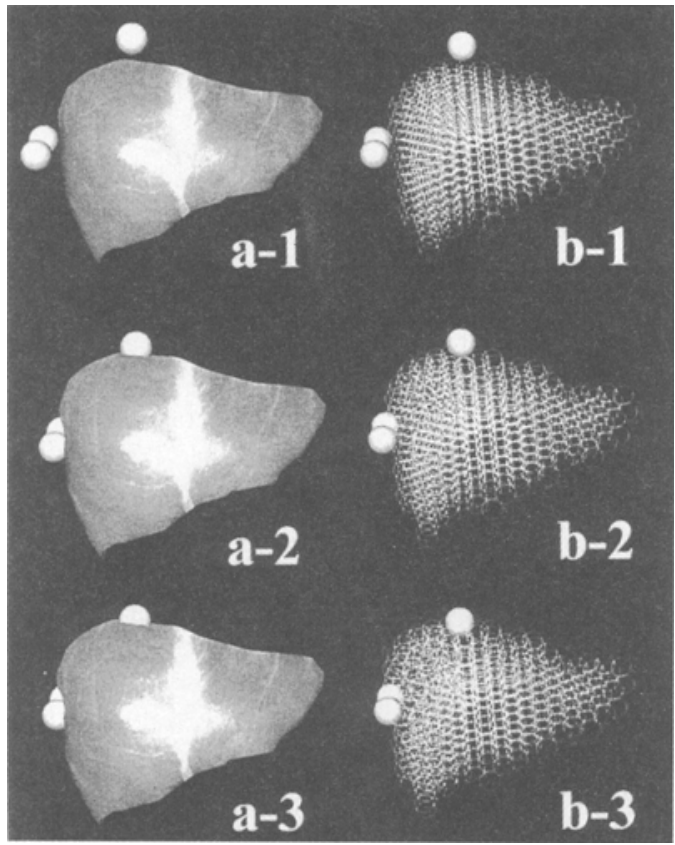

Fig.5. The response of the deformable liver model to variations in force. Fig. 5a-1 to a-3 shows the performance of the model when it was pushed by three fingers. Fig. 5b-1 to b-3 show the condition of spheres of the model.

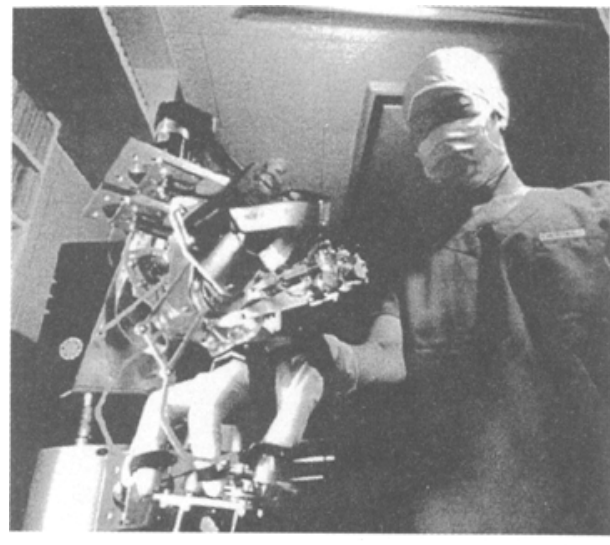

a

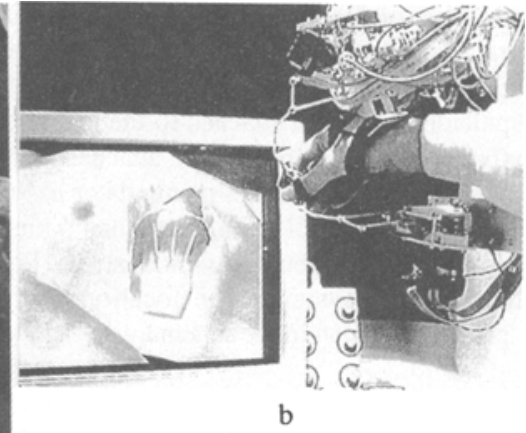

Fig.6. The appearance of the force feedback system .The operator's thumb, forefinger and middle finger are attached to the distal part of the motion controlled manipulators. 


\section{Results}

Fig. 1 shows a typical scene of the user performing a surgical simulation with our system. In this image the 3D image which the user observes with a head mount display (HMD) is composited to clear the user's works. In this case the user is not using the force feedback system which we developed, and is instead using a cyberglobe which is commercially available. Using the liver as an example, the structure of our deformable organ model is shown in Fig. $2 a, 2 b$ and $2 c$. Fig. $2 a$ and $2 b$ are the reconstructed liver model by the sphere filled model. The liver contours are obtained from the 3D data set of MRI with a $4 \mathrm{~mm}$ slice in a $4 \mathrm{~mm}$ pitch and 1880 spheres fill this liver model. The surface of the liver model was wrapped with surface images of autopsied liver tissue using a texture mapping method to obtain a realistic appearance for the simulation (Fig.2b). In this simulation the surface texture can be easily changed to color code by the user (Fig.2a). Fig. 2c shows the spheres that fill the liver. In this image the spheres were displayed with the wire frame image and the liver surface were eliminated.Fig. 3 show the basic behavior of model deformation. If external force is applied to the object, each element sphere is pressed and moved in tum (Fig. $\left.3 b^{\prime}\right)$. The entire object is deformed (Fig.3b). Fig. 5 show the response of the deformable liver model to variations in force. Fig. 5a-1 to a-3 shows the performance of the model when it was pushed by three fingers. The white ball indicates the object that pushed the surface of the model liver. Fig. 5b-1 to b-3 show the condition of spheres of the model. These images were displayed at 10-12 frames/s. And there was no significant difference in delay in image generation. Fig. $6 \mathrm{a}, \mathrm{b}$ shows the appearance of the force feedback system. A surgeon attached his right arm to the device. The operator's thumb, forefinger and middle finger are attached to the distal part of the motion controlled manipulators. By the combination of sphere-filled model and force feedback device, it was possible to manipulate a model liver with 1880 spheres at a speed of about 10 frame/s. The rate of image generation is about 10 fold slower than the responsiveness of the force feedback device. We had to interpolate the magnitude of force when it was supplied to the force feedback device in order to create a realistic sense of touch.

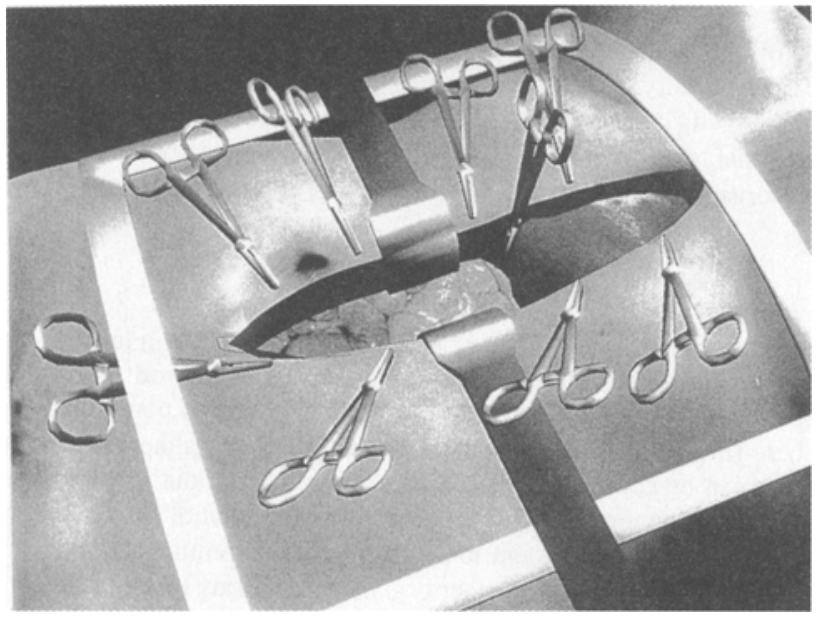

Fig.7. A simulation of open surgery in the abdominal region. Forceps, abdominal retractor and surgical knife are used in the simulation. 


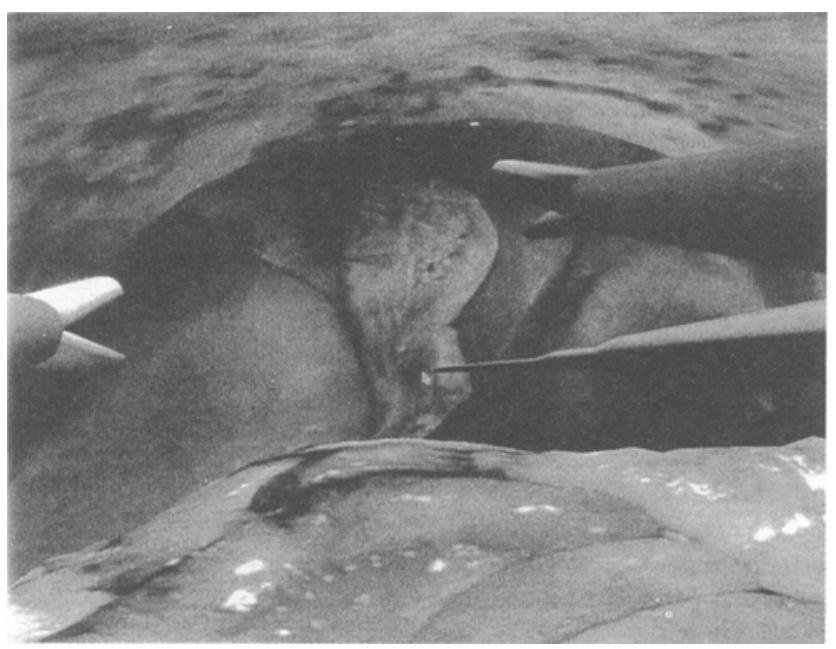

Fig.8. A simulation of laparoscopic surgery in the abdominal region with the same data set used for the open surgery in Fig.7.

In this image the 3D image which the operator observes with a head mount display (HMD) is composited on the image of the user.

This speed was not changed when the operator touched the organ model with one finger or three fingers. One of the benefits of the sphere-filled model is that there is no difference in calculation time when the number of outer forces is different. Fig. 7 shows a simulation of open surgery in the abdominal region. Forceps, abdominal retractor and surgical knife are used in this simulation. Data from the patient was reconstructed from the MRI data set and the skin and organ surfaces were texture mapped (skin surface, patient's surface image; liver and intestines, Image of autopsied specimen) to increase the reality in the virtual environment. When the view point, field of vision and surgical tools are changed, laparoscopic surgery can be performed. Fig. 8 shows laparoscopic surgery in the abdominal region with the same data set used for the open surgery in Fig. 7. It is possible to incise the organ surface with an electric knife and to pinch the liver with forceps.

\section{Conclusion}

By filling the object model with element spheres, containing a relatively with high speed manipulation for controlling deformation was achieved. This method is suitable for real-time simulation and quantitative deformation of tissues. We are also albe to point out a few merits of this method. In the sphere-filled model, no matter how complicated the shapes of the organ of interest may be, organ models can be constructed by the same operation. In our virtual surgery system, it is possible to perform surgical maneuvers at a speed approaching that of the actual procedure, with a sense of touch. We have applied this system to surgery in the abdominal region in this paper and we plan to extend the region of application to other organs and various types of surgery. This surgical experience, in which a patient's morphological characteristics are reproduced in a virtual cnvironment, will allow the establishment of a new method of clinically applicable surgery as well as an cducational innovation, in the near future. 


\section{References}

1. Thomas H. Speeter: Three Dimensional Finite Eement Analysis of Eastic Continut for Tactile Sensing: The international Jounal of Robotics Reserch, 11 No. 1:1-19.February 1992.

2. Robb, R.A, D.P. Hanson: The ANALYZE software system for vistalization and analysis in surgery simulation. In: Computer Integrated Surgery, Eds. Steve Lavalle, Russ Taylor, Greg Burdea and Ralph Mosges, MIT Press, 1993.

3. Stzuki. N. Takatsu. A, Kita. K. Tanaka T, Inaba. R, Fukui. K. Development of an 3D image simulation system for organ and soft tissue operations.: Abstract of the World Congress on Medical Physics and Biomedical Engineering 1994; 39a: 609 .

4. Suzuki. N, Hattori. A, Takatsu A: Medical vintual reality system for surgical planning and surgi cal support.: J. Comput. Aided Surg., 54-59, 1(2), 1995

5. Robb, R.A, D.P. Hanson, J.J. Camp: Computer-aided surgery planning and rehearsal at Mayo Clinic. Computer, 29(1):39-47, 1996.

6. Suzuki. N, Kawakami. K, Hattori. A. Takatsu A.: Abdominal surgery planning using medical vintual reality system.: CAR'96 1996; 1058

7. S.Cotin, H. Delingette, M. Bro-Nielsen, N. Ayache, J.M. Clement, V. Tassetti, J. Marescaux: Geometric and Physical Representations for a Simulator of Hepatic Surgery: Health Care in the Information Age, Eds:H.Sieburg. S. Weghorst, K.Morgan, IOS Press and Ohmsha, 1996.

8. Dwight A. Meglan, Rakesh Raju, Gregory L Merril, Jonathan R. Merril, Binh H. Nguyen, Shankar N. Swamy, Gerald A. Higgins: The Teleos Virtual Environment Toolkit for Simulation-Based Surgical Eoucation: Health Care in the Information Age, Eds:H.Sieburg, S. Weghorst, K.Morgan, 10 S Press and Ohmsha, 1996.

9. Naoki Suzuki, Asaki Haltori. Shinya Kai, Akithiro Takatsu: Surgical planning system for solt tissues using virtual reality: In: Medicine Meets Virtual Reality, Eds: K.S.Morgan et al. .pp. 159-163, IOS Press, 1997. 